\title{
Jie-du granule preparation for the treatment of advanced hepatocellular carcinoma: a retrospective cohort study of 177 patients
}

\author{
L.Y. Chen ${ }^{1}$, X.F. Zhai ${ }^{1,}{ }^{*}$, Z. Chen ${ }^{1}$, J.F. Zhu ${ }^{2}$, P.A. Qian², H.T. Zhao ${ }^{1}$ and C.Q. Ling ${ }^{1}$ \\ ${ }^{1}$ Department of Traditional Chinese Medicine, Changhai Hospital, Second Military Medical University, Shanghai, China \\ 2 Department of Liver Disease, Shanghai Municipal Hospital of Traditional Chinese Medicine, Shanghai, China \\ * These authors have contributted equally to this work \\ Correspondence to: C.Q. Ling, email: changquanling@smmu.edu.cn \\ Keywords: hepatocellular carcinoma, Jie-du granule, best supportive treatment, retrospective cohort study \\ Received: July 28, $2016 \quad$ Accepted: October 19, $2016 \quad$ Published: October 25, 2016
}

Copyright: Chen et al. This is an open-access article distributed under the terms of the Creative Commons Attribution License (CC-BY), which permits unrestricted use, distribution, and reproduction in any medium, provided the original author and source are credited.

\section{ABSTRACT}

Objective: To compare the clinical efficacy of Jie-du granule preparation versus best supportive treatment in patients with advanced hepatocellular carcinoma.

Methods: A retrospective cohort study was carried out in patients with advanced liver cancer. Patients were divided into Jie-du granule treatment (JD) and best supportive treatment (BST) groups. The main outcomes included median overall survival time.

Results: A total of 177 patients with Barcelona Clinic Liver Cancer stage C receiving JD granule treatment or BST were enrolled between January 2012 and December 2014. The overall median survival time was 6.2 months (95\% confidence interval [CI] 4.546-7.854) in the JD group versus 4 months (95\% CI 3.471-4.529) in the BST group. Significant independent risk factors were alpha-fetoprotein $(P=$ $0.048)$, Child-Pugh class $(P=0.005)$, vascular invasion $(P=0.003)$, and extrahepatic metastasis $(P=\mathbf{0 . 0 0 1 8})$. For patients with two or fewer of these independent risk factors, the overall median survival of those treated with JD was significantly longer than that of patients receiving BST $(P<0.05)$.

Conclusion: Jie-du granule preparation may prolong survival of patients with advanced HCC.

\section{INTRODUCTION}

Many patients with hepatocellular carcinoma (HCC) in China are diagnosed with Barcelona Clinic Liver Cancer stage C (BCLC-C) disease. Sorafenib and best supportive treatment (BST) have been recommended as standard treatments for HCC patients staged as BCLC-C [1]. However, sorafenib is not widely used in clinical practice in China because it is expensive. Instead, many HCC patients with advanced cancer stage and/or poor liver functional reserve receive traditional Chinese medicine (TCM) treatment. Jie-du granule preparation is a commonly used Chinese herbal medicine formula for HCC [2-3]. Under the supervision of Professor CQ Ling and Professor JF Zhu, more than 20,000 HCC patients have received JD granule treatment from 1996 to 2016.
However, few studies have focused on the therapeutic efficacy of Jie-du granule for BCLC-C HCC, raising the question of whether treatment with Jie-du granule alone might be superior to best supportive treatment. Therefore, we conducted a retrospective cohort study to (i) compare the efficacy of Jie-du granule and best supportive treatment, and (ii) evaluate whether TCM alone is an effective treatment approach for advanced HCC.

\section{MATERIALS AND METHODS}

Study population

A consecutive case series of HCC patients treated with Jie-du granule or best supportive treatment at 
Table 1: Patient baseline and clinical characteristics $(n=177)$

\begin{tabular}{|c|c|c|c|c|}
\hline & & JD & BST & $\mathbf{P}$ \\
\hline Age, y & & $58.4 \pm 12$ & $60.3 \pm 14.5$ & 0.34 \\
\hline \multirow[t]{2}{*}{ Sex } & Male & 67 & 78 & \\
\hline & Female & 12 & 20 & 0.37 \\
\hline \multirow[t]{2}{*}{ Child-Pugh } & A & 32 & 26 & \\
\hline & B & 47 & 71 & 0.05 \\
\hline \multicolumn{5}{|l|}{ Etiology } \\
\hline \multirow[t]{2}{*}{ HBV-Ag } & - & 12 & 13 & \\
\hline & + & 67 & 84 & 0.73 \\
\hline \multicolumn{5}{|l|}{ Disease burden } \\
\hline \multirow[t]{2}{*}{ Lymphatic metastasis } & - & 36 & 53 & \\
\hline & + & 43 & 45 & 0.53 \\
\hline \multirow[t]{2}{*}{ Vascular invasion } & - & 33 & 31 & \\
\hline & + & 46 & 67 & 0.16 \\
\hline Main branch & & 17 & 10 & \\
\hline Left branch & & 21 & 10 & \\
\hline Right branch & & 16 & 18 & \\
\hline Other & & 3 & 8 & 0.11 \\
\hline \multirow[t]{2}{*}{ Extrahepatic metastasis } & - & 52 & 53 & \\
\hline & + & 27 & 45 & 0.07 \\
\hline Tumor size & $<5 \mathrm{~cm}$ & 37 & 53 & \\
\hline \multirow{2}{*}{ Tumor number } & $\geq 5 \mathrm{~cm}$ & 42 & 45 & 0.55 \\
\hline & Single & 8 & 10 & \\
\hline \multirow{5}{*}{ Tumor location } & Multiple & 59 & 67 & \\
\hline & Diffuse & 4 & 12 & 0.25 \\
\hline & Left lobe & 9 & 49 & \\
\hline & Right lobe & 23 & 10 & \\
\hline & Diffuse & 12 & 18 & 0.41 \\
\hline
\end{tabular}

Changhai Hospital and Shanghai Traditional Chinese Medicine Hospital from January 2012 to December 2014 was studied retrospectively. The inclusion criteria for the study population were as follows: (i) HCC diagnosed according to the criteria issued by the European Association for the Study of Liver Disease/American Association for the Study of Liver Disease [4]; (ii) confirmed BCLC-C; (iii) patients received only traditional Chinese treatment, of which Jie-du granule preparation was dominant (only received treatment with oral JD guanule preparation), or only best supportive treatment. The exclusion criteria were as follows: (i) severe disease or dysfunction of heart, brain, or kidney; (ii) receiving other anticancer treatment after enrollment; (iii) second primary malignancy. The enrolled patients were divided into the JD (Jie-du granule) group and the BST (best supportive treatment) group depending on the treatment received. The study protocol was approved by the ethics committees of all participating hospitals. Collection of informed consent was waived given the retrospective nature of the study.

\section{Medical treatment}

All patients in the JD group received only Jie-du granule preparation (produced by the Good Manufacturing Practice-certified Tianjiang Pharmaceutical Factory, Jiangsu, China; Production License No. Su $\mathrm{ZzY} 20010266$ ), at an oral dose of $8 \mathrm{~g}$ (equal to $80 \mathrm{~g}$ of raw herbal material) twice a day, 30 minutes after a meal. The JD granule preparation is composed of four traditional Chinese herbal medicines, root of Actinidia valvata, root of Salvia chinensis, bulb of Cremastra appendiculata, and gizzard membrane of Gallus gallus domesticus, which are extracted by hot water and lyophilization and are 
Table 2: Predictors of overall survival in $177 \mathrm{HCC}$ patients

\begin{tabular}{|c|c|c|c|c|c|c|}
\hline \multirow[t]{2}{*}{ Variable } & \multicolumn{3}{|c|}{ Univariate analysis } & \multicolumn{3}{|c|}{ Multivariate analysis } \\
\hline & HR & $95 \% \mathrm{CI}$ & $\boldsymbol{P}$ & HR & $95 \%$ CI & $\boldsymbol{P}$ \\
\hline Child-Pugh(B/A) & 1.698 & $1.223-2.358$ & 0.002 & 1.669 & $1.172-2.378$ & 0.005 \\
\hline $\operatorname{AFP}(\geq 400 /<400 \mathrm{ng} / \mathrm{dl})$ & 1.264 & $0.934-1.709$ & 0.129 & 1.385 & $1.003-1.913$ & 0.048 \\
\hline Vascular invasion(yes/no) & 1.471 & $1.074-2.017$ & 0.016 & 1.706 & $1.201-2.423$ & 0.003 \\
\hline $\begin{array}{l}\text { Extrahepatic } \\
\text { metastasis(yes/no) }\end{array}$ & 1.370 & $1.009-1.860$ & 0.044 & 1.516 & $1.075-2.137$ & 0.018 \\
\hline Treatment(JD /BST ) & 1.791 & $1.310-2.448$ & 0.000 & 1.399 & $1.001-1.956$ & 0.050 \\
\hline Gender(female /male) & 0.977 & $0.663-1.440$ & 0.907 & & & \\
\hline Age & 0.987 & $0.977-0.998$ & 0.022 & & & \\
\hline $\begin{array}{l}\text { Lymphatic } \\
\text { (yes/no) }\end{array}$ & 1.024 & $0.758-1.385$ & 0.875 & & & \\
\hline $\mathrm{HBV}-\mathrm{Ag}(+/-)$ & 0.860 & $0.564-1.312$ & 0.484 & & & \\
\hline Tumor size $(\geq 10 /<10 \mathrm{~cm})$ & 1.010 & $0.741-1.378$ & 0.949 & & & \\
\hline
\end{tabular}

present in a proportion of 1:1:0.4:0.4 [4]. All patients in the BST group received best supportive therapies selected according to the preferred practices at each center.

\section{Follow-up evaluation}

The patients were followed up by telephone and the time of death was recorded. The end of the followup period was either death or May 2015. The primary endpoint was overall survival (OS), which was measured from the time of initial treatment in the study until the time of death or May 2015.

\section{Statistical analysis}

Survival was assessed according to the KaplanMeier method, and curves were compared using the logrank test. The Cox proportional hazard model was used to assess the prognostic values of the variables. The following parameters were selected: age, gender, etiology, $\alpha$-fetoprotein (AFP), extrahepatic metastasis, lymphatic metastasis, vascular invasion, tumor size, and Child-Pugh classification. Statistical analyses were carried out using SPSS software, version 20.0 (SPSS, Inc., Chicago, IL).

\section{RESULTS}

\section{Patient characteristics}

A total of 194 BCLC-C stage HCC patients were enrolled from January 2012 to December 2014. We excluded 17 patients whose family members did not cooperate in the follow-up, therefore 177 patients who met the inclusion criteria were finally evaluated. Of these patients, 79 were in the JD group and 98 in the BST group. Most patients in the JD group only received long-term maintenance treatment with oral JD granule preparation. Only 3 patients received other herbal medicine treatment (no more than 4 kinds of herbals) because of fatigue, abdominal distension and other symptoms. The two groups were comparable in age, sex, Child-Pugh, disease burden, etiology, and AFP. Detailed baseline clinical characteristics of these 177 patients are summarized in Table 1.

\section{Survival of patients}

Of the 79 patients in the JD group, 5 patients survived and the remaining 74 patients died. All 98 patients in the BST group died. The median OS time in the JD group was 6.2 months $(95 \%$ confidence interval [CI], 4.379-8.221) compared with 4 months (95\% CI, 3.471-4.529) in the BST group. The log-rank analysis showed that the median survival time in the JD group was significantly longer than that in the BST group $(P<0.001)$ (Figure 1).

\section{Prognostic analysis}

In the multivariate Cox model, the following four parameters were considered as risk factors: Child-Pugh B class, AFP $\geq 400 \mathrm{ng} / \mathrm{dL}$, vascular invasion, and extrahepatic metastasis, with corresponding hazard ratios (HR) of 1.669 (95\% CI 1.172-2.378), 1.385 (95\% CI 1.003-1.913), 1.706 (95\% CI 1.201-2.423), and 1.516 (95\% CI 1.075-2.137) respectively. These variables were independent prognostic risk factors (Table 2). 


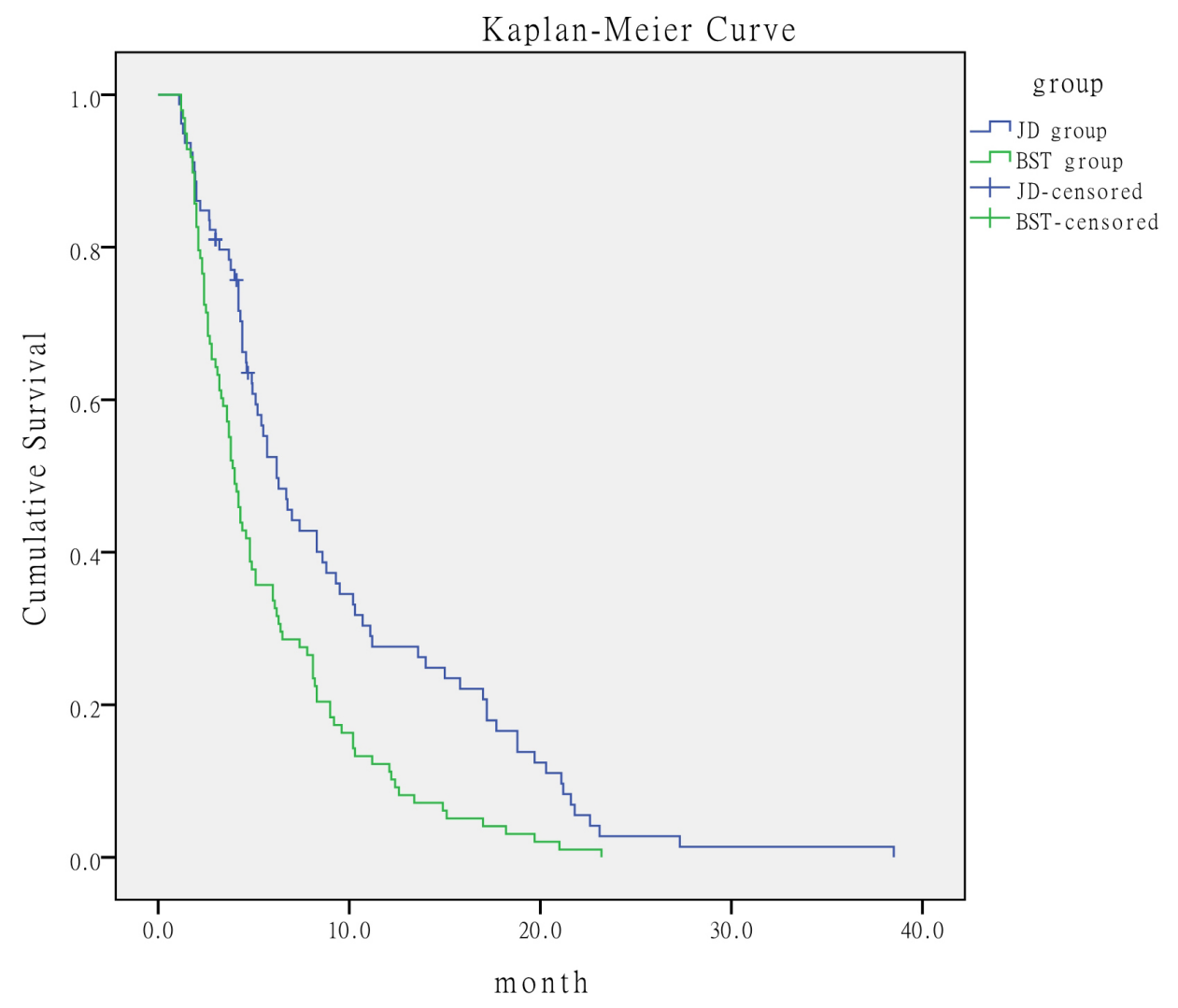

Figure 1: Comparison of survival time of patients according to treatment.

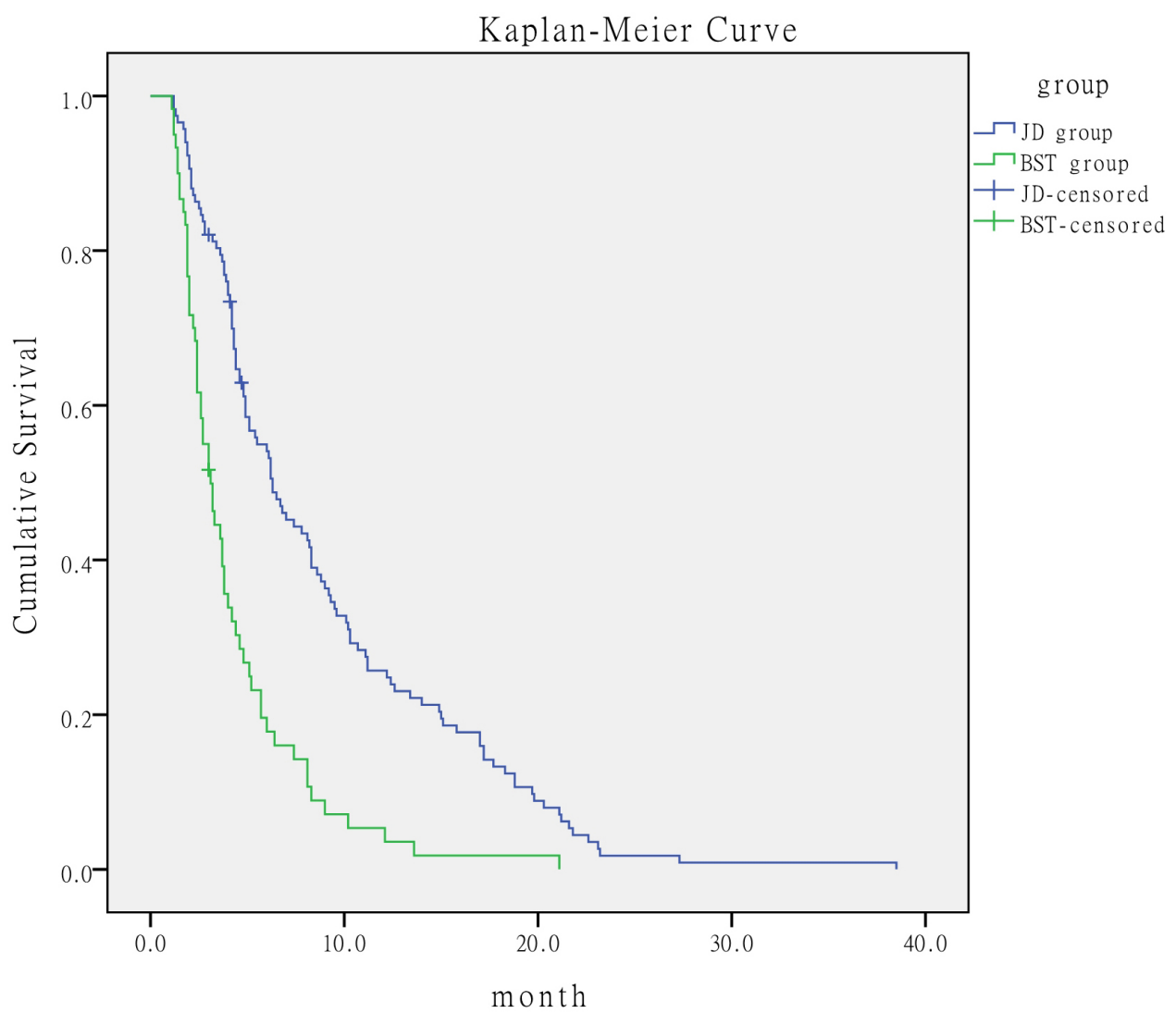

Figure 2: Comparison of survival time of patients with $\leq 2$ prognostic risk factors according to treatment. 


\section{BCLC-C stage subgroups}

We subdivided the BCLC-C stage into four subgroups according to the presence of independent prognostic risk factors of Child-Pugh, AFP, vascular invasion, and extrahepatic metastasis as follows: no or 1 prognostic risk factor, 2 prognostic risk factors, 3 prognostic risk factors, and 4 prognostic risk factors. Patients with 0-2 prognostic risk factors in the JD group had better outcome than the corresponding patients in the BST group. The median survival time was 8.8 months (95\% CI, 5.570-12.030) in the JD group versus 4.6 months (95\% CI, 3.835-5.365) in the BST group. The log-rank test showed that the median survival for the JD group was significantly longer than that for the BST group ( $P$ $<0.001$ ) (Figure 2). When the number of prognostic risk factors ranged from 3 to 4 , the median survival time was 3.2 months (95\% CI, 1.794-4.606) in the JD group versus 3.1 months (95\% CI, 2.266-3.934) in the BST group, with no significant difference between the two groups $(P=$ 0.659) (Figure 3).

\section{DISCUSSION}

Sorafenib and supportive treatment are recommended as standard treatments for HCC by the
American Association for the Study of Liver Diseases (ASSLD). In the clinical trial of sorafenib in the AsiaPacific region (ORIENTAL), median OS in the sorafenib group was 6.5 months compared with 4.2 months in the placebo group [5]. In our study, the median OS time was 6.2 months (95\% CI, 4.379-8.221) in the JD group versus 4 months $(95 \% \mathrm{CI}, 3.471-4.529)$ in the BST group, which was similar to the results of the ORIENTAL study. This result suggested that the efficacy of Jie-du granule preparation might be similar to that of sorafenib. Both treatments could prolong survival of patients with advanced HCC and both showed better efficacy than supportive treatment. However, the price of the Chinese herb is much lower than that of sorafenib.

The patients were divided into four subgroups based on 4 independent prognostic risk factors (Child-Pugh, AFP, vascular invasion, and extrahepatic metastasis). The comparison of survival time of patients in different subgroups demonstrated the heterogeneity of BCLC-C HCC patients, and further showed that patients with vascular invasion, extrahepatic metastasis, or poor liver function had higher mortality than those without these prognostic risk factors. Therefore, a single therapy mode for all patients might not be appropriate. We conclude that patients with two or fewer of these prognostic risk factors should receive traditional Chinese medicine treatment rather than best supportive treatment. Moreover, our data

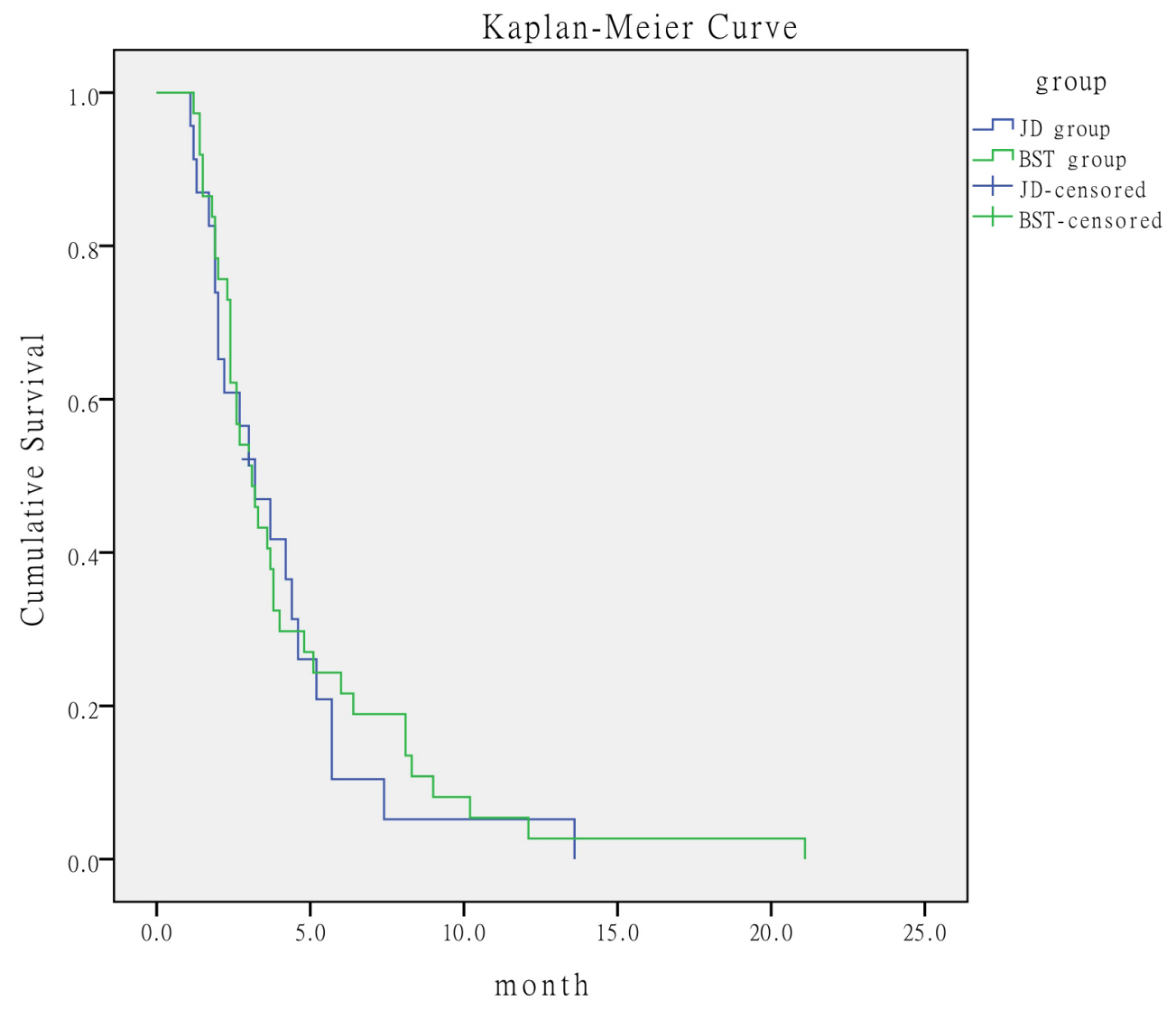

Figure 3: Comparison of survival time of patients with $\geq 3$ prognostic risk factors according to treatment. 
strongly suggest that stratification of BCLC-C is of great significance for optimal treatment of advanced HCC.

Jie-du granule preparation is composed of Salvia chinensis Benth, root of Actinidia valvata, bulb of Cremastra appendiculata, and ventriculi galli mucosa [6]. Previous trials have demonstrated that these cancerfighting Chinese herbs inhibited development of liver cancer $[2,3,7,8]$. Shim and Xin proved that Salvia chinensis Benth and bulb of Cremastra appendiculata showed effective antiangiogenesis and cytotoxic activity in vitro against the BEL-7402 and SMMC-7721 tumor cell lines [9]. Zhang demonstrated that the active fraction from Actinidia valvata had an inhibitory effect on transplanted H22 mouse tumor cells [10]. The underlying mechanism may be through modulation of the cell cycle and induction of cellular apoptosis.

The results of our study demonstrated that the traditional Chinese medicine prolonged survival of patients with advanced HCC. Moreover, the median survival time of patients who received Jie-du granule was similar to that of patients who received sorafenib, suggesting that the efficacy of Jie-du granule is probably similar to that of sorafenib. A randomized prospective trial has been conducted to compare the efficacy of Jiedu granule and sorafenib. The results of this trial will be revealed at the end of 2016.

\section{ACKNOWLEDGMENTS}

The study was supported by Shanghai Municipal Commission of Health and Family Planning (ZY3LCPT-2-1004) and E-institutes of Shanghai Municipal Education Commission (E 03008).

\section{COMPETING INTERESTS} interests.

The authors declare that they have no competing

\section{Authors' contributions}

LY Chen and XF Zhai contributed equally to this work.

\section{REFERENCES}

1. Bolondi L, Burroughs A, Dufour JF, Galle PR, Mazzaferro V, Piscaglia F, Raoul JL, Sangro B. Heterogeneity of patients with intermediate (BCLC-B) hepatocellular carcinoma: proposal for a subclassification to facilitate treatment decisions. Semin Liver Dis. 2012; 32:348-9. doi: $10.1055 / \mathrm{s}-0032-1329906$
2. Ying ZF, Zheng GY, Yao M. The Experience of Professor Ling Chang-quan on the Treatment of Malignant Tumor. Guid J Tradit Chin Med Pharm. 2015; 21: 17-21. doi: 10.13862/j.cnki.cn43-1446/r.2015.02.006

3. Yu Y, Lang Q, Chen Z, Li B, Yu C, Zhu D, Zhai X, Ling C. The efficacy for unresectable hepatocellular carcinoma may be improved by transcatheter arterial chemoembolization in combination with a traditional Chinese herbal medicine formula. Cancer. 2009; 115: 5132-8. doi: 10.1002/ cncr.24567

4. Bruix J, Sherman M, Llovet JM, Beaugrand M, Lencioni R, Burroughs AK, Christensen E, Pagliaro L, Colombo M, Rodés J; EASL Panel of Experts on HCC. Clinical management of hepatocellular carcinoma. Conclusions of the Barcelona-2000 EASL conference. European Association for the Study of the Liver. J Hepatol. 2001; 35: 421-30. http://linkinghub.elsevier.com/retrieve/pii/ S0168827802003148

5. Cheng AL, Kang YK, Chen Z, Tsao CJ, Qin S, Kim JS, Luo R, Feng J, Ye S, Yang TS, Xu J, Sun Y, Liang H, et al. Efficacy and safety of sorafenib in patients in the AsiaPacific region with advanced hepatocellular carcinoma: a phase III randomised, double-blind, placebo-controlled trial. Lancet Oncol. 2009; 10: 25-34. doi: 10.1016/S14702045(08)70285-7

6. Ling CQ. Cancerous toxin is the key pathogenic factor of malignant tumor. J Chin Integr Med. 2008; 6: 111-114. doi: 10.3736/jcim20080201

7. Wang X, Wang N, Cheung F, Lao L, Li C, Feng Y. Chinese medicines for prevention and treatment of human hepatocellular carcinoma: current progress on pharmacological actions and mechanisms. J Integr Med. 2015; 13: 142-64. doi: 10.1016/S2095-4964(15)60171-6

8. Zhai XF, Chen Z, Li B, Shen F, Fan J, Zhou WP, Yang YK, Xu J, Qin X, Li LQ, Ling CQ. Traditional herbal medicine in preventing recurrence after resection of small hepatocellular carcinoma: a multicenter randomized controlled trial. J Integr Med. 2013; 11: 90-100. doi: 10.3736/jintegrmed2013021

9. Shim JS, Kim JH, Lee J, Kim SN, Kwon HJ. Antiangiogenic activity of a homoisoflavanone from Cremastra appendiculata. Planta Med. 2004; 70:171-173. doi: 10.1055/ s-2004-815496

10. Zhang YN, Liu L, Ling CQ. Inhibition effect of active fraction from Actinidia valvata on growth of transplanated mouse tumor cells and preliminary study of its mechanism. Chin J Chin Mater Med. 2006; 31: 918-920. http://www. cnki.com.cn/Article/CJFDTOTAL-ZGZY200611015.htm 